(C) [2009] IEEE. Reprinted, with permission, from Dorrell, David; Hsieh, Min-Fu; Guo, Youguang. 2009, 'Unbalanced Magnet Pull in Large Brushless Rare-Earth Permanent Magnet Motor with Rotor Eccentricity', IEEE Transactions On Magnetics, Vol. 45 , no. 10 , pp. 4586-4589. This material is posted here with permission of the IEEE. Such permission of the IEEE does not in any way imply IEEE endorsement of any of the University of Technology, Sydney's products or services. Internal or personal use of this material is permitted. However, permission to reprint/republish this material for advertising or promotional purposes or for creating new collective works for resale or redistribution must be obtained from the IEEE by writing to pubspermissions@ieee.org. By choosing to view this document, you agree to all provisions of the copyright laws protecting it. 


\title{
Unbalanced Magnet Pull in Large Brushless Rare-Earth Permanent Magnet Motors With Rotor Eccentricity
}

\author{
David G. Dorrell ${ }^{3}$, Min-Fu Hsieh², and YouGuang Guo ${ }^{3}$ \\ ${ }^{1}$ School of Mechanical, Electrical and Mechatronic Systems, University of Technology Sydney N.S.W. 2007, Australia \\ 2 Department of System and Navel Mechatronic Engineering and Research Center of Ocean Environment and Technology, \\ National Cheng Kung University, Tainan 701, Taiwan
}

\begin{abstract}
Permanent magnet motors are now the focus of application in larger drive and generator systems. They often utilize rare-earth magnets where attractive forces are large and unbalanced magnetic pull (UMP) will be generated even when unexcited. In this paper, a 4-pole machine design is utilized which has either surface magnets or consequent poles. Dynamic eccentricity up to $80 \%$ is put into the machine model and a variety of simulations carried out to investigate the UMP. It is found that with strong and thick magnets the machine is robust and the UMP is almost load independent. The consequent pole rotor arrangement produces much higher UMP when the dynamic eccentricity aligns with the steel poles. In the simulations, the different stress components are investigated to assess the validity of a commonly held approximation where the radial force is taken to be a function of the square of the radial air-gap flux.
\end{abstract}

Index Terms - Permanent magnet motors, unbalanced magnetic pull (UMP).

\section{INTRODUCTION}

$\mathbf{U}$ BALANCED MAGNETIC PULL (UMP) is usually associated with larger machines [1], [2], [12]-[14] or other types of machine [15], [16]. Small permanent magnet (PM) machines are subject to UMP [3] even without rotor anomalies. UMP is important because it affects bearing wear, noise, and vibration [4], [5]. There is surprisingly little literature on UMP in PM machines; in [6], several fractional-slot ferrite-magnet motors (9-slot/8-pole machines and 18-slot stator with 12-pole/16pole/20-pole rotor machines) were investigated — these had surface magnet and consequent pole rotors and considered static rotor eccentricity. Dorrell et al. [6] and Li et al. [17] developed analytical UMP calculations for UMP in PM machines but saturation can lead to inaccuracies [2]. Dynamic rotor eccentricity is addressed in this paper in a higher power 18-slot/4-pole machine with rare-earth surface-mounted magnets and consequent-pole rotors. The equations for the UMP and stresses are implemented using 2-D finite element solutions and investigate the component values of the stress terms and how they vary with load and rotor eccentricity.

\section{Calculation of Unbalanced Magnetic Pull}

There is literature on UMP, both in terms of analytical calculation and finite element analysis [1], [3]. UMP is caused by flux concentration in the air gap. Smith and Dorrell [8] show that this situation is generated by flux waves with pole pairs differing by one. Air-gap permeance modulation can be used to derive eccentricity-generated air-gap flux waves [8]. For a three-phase fractional-slot winding, the MMF is formed from a fundamental pole number with 5th, 7th, 11th, 13th, etc., harmonics. Hence, the radial air-gap flux is

$$
\begin{aligned}
b_{s}(y, t) & =\sum_{n} B_{s}^{n} \cos \left(\omega_{s} t-n k y+\phi_{n}\right) \\
& =\operatorname{Re} \sum_{n} \frac{\bar{B}_{s}^{n}}{2} e^{j\left(p \omega_{r} t-n k y\right)}
\end{aligned}
$$

Corresponding author: D. G. Dorrell (e-mail: ddorrell@eng.uts.edu. au). where $\omega_{s}$ is the current frequency (rad/s), $\omega_{r}$ is the rotational speed, $p$ is the pole-pair number of the rotor, $y$ is the tangential direction (counterclockwise), and $k$ is the inverse of the mean air-gap radius. $n=N_{w},-5 N_{w}, 7 N_{w}$, etc. If there is dynamic rotor eccentricity, the stator flux waves are

$$
\begin{aligned}
b_{s}(y, t)=\operatorname{Re} \sum_{n}\left(\frac{\bar{B}_{s}^{n}}{2} e^{j\left(p \omega_{r} t-n k y\right)}\right. & \\
& \left.+\frac{\lambda_{e c c} \bar{B}_{s}^{n}}{4} e^{j\left(\omega_{r}(p \pm 1) t-(n \pm 1) k y\right)}\right) .
\end{aligned}
$$

The permeance factor $\lambda_{e c c}$ is a function of the air-gap length, degree of dynamic eccentricity (rotation on center of stator bore but off-center of rotor axis), and in the case of a PM machine, the magnet thickness. For the PM rotor, we get

$$
\begin{aligned}
b_{r}(y, t)=\operatorname{Re} \sum_{m}( & \frac{\bar{B}_{r}^{m}}{2} e^{j m\left(\omega_{r} t-p k y\right)} \\
& \left.+\frac{\lambda_{e c c} \bar{B}_{r}^{m}}{4} e^{j\left((m \pm 1) \omega_{r} t-(m p \pm 1) k y\right)}\right)
\end{aligned}
$$

Both (2) and (3) produce flux waves with pole pairs differing by one; however, the rotating speed of the waves will differ, which will lead to forces that are either steady radial pulls or vibrations. Let us consider two general flux waves and the approximate normal Maxwell stress in the air gap. The standard approximation considers only the radial flux

$$
\begin{aligned}
& \sigma= \frac{b_{n}^{2}}{2 \mu_{0}} \\
&=\frac{1}{2 \mu_{0}} \operatorname{Re}\left[\bar{B}_{n} e^{j\left(\omega_{n} t-n k y\right)}\right] \times \operatorname{Re}\left[\bar{B}_{m} e^{j\left(\omega_{m} t-m k y\right)}\right] \\
&=\frac{1}{4 \mu_{0}} \operatorname{Re}\left[\bar{B}_{n} \bar{B}_{m} e^{j\left(\left(\omega_{n}+\omega_{m}\right) t-(n+m) k y\right)}\right. \\
&\left.+\bar{B}_{n} \bar{B}_{m}^{*} e^{j\left(\left(\omega_{n}-\omega_{m}\right) t-(n-m) k y\right)}\right] .
\end{aligned}
$$

In many publications, this is worked through to obtain expressions for the radial force; in this paper, the UMP is obtained from 2-D finite element analysis. Binns and Dye [9] suggested that the tangential flux density components are small but they still generate up to a $10 \%$ of the UMP. The normal and tangential stresses are

$$
\sigma=\frac{b_{n}^{2}-b_{t}^{2}}{2 \mu_{0}} \quad \text { and } \quad \gamma=\frac{b_{n} b_{t}}{\mu_{0}}
$$




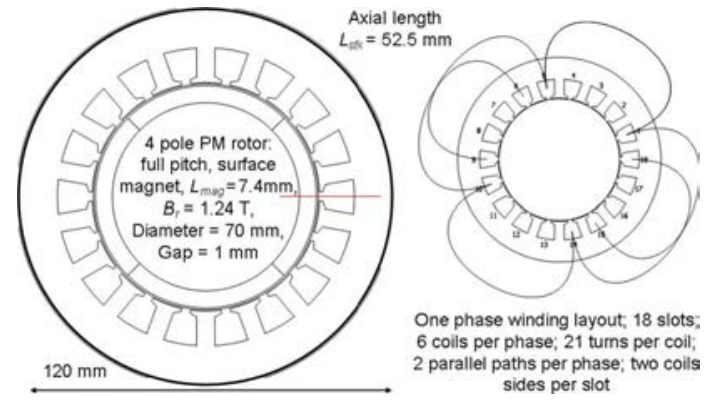

Fig. 1. Basic machine topology for surface-magnet 4-pole machine.

where $b_{t}$ is positive in the counterclockwise direction and $b_{n}$ is positive when flowing out in the radial direction. These can be used to calculate the UMP in orthogonal directions by taking a stress integral around the air gap so that

$$
\begin{aligned}
& F_{d}=L \int_{0}^{2 \pi r}(\sigma \cos (k y)-\gamma \sin (k y)) d y \\
& F_{q}=L \int_{0}^{2 \pi r}(\sigma \sin (k y)+\gamma \cos (k y)) d y
\end{aligned}
$$

where $L$ is the axial core length. Finite element analyses were carried out and equations are implemented in a stepwise fashion. The shear stress components are usually ignored so an opportunity is taken to validate (4) as a good approximation.

\section{MACHINE SPECIFICATION}

The machine cross section and phase winding layout are given in Fig. 1 with some basic properties and several geometrical parameters. The machine weighs about $4.7 \mathrm{~kg}$ in total which is useful in terms of comparison to the UMP force. The machine is designed for $6000 \mathrm{r} / \mathrm{min}$ operation which gives a power rating of just over $6 \mathrm{KW}(10.3 \mathrm{Nm}$ of torque). This machine can operate under either alternating current (ac) (sine-wave) or direct current (dc) (square-wave) control since the back-EMF is intermediate between sine and trapezoidal shape. Fig. 2 shows the current (phase 1), back-EMF, and torque when the current is on the $q$-axis for both sine-wave and square-wave operation. This was obtained from SPEED PC-BDC (University of Glasgow, U.K.). Because the back-EMF is not sinusoidal, then there is torque ripple when sinusoidal current is fed into the machine. In the simulations, sine-wave operation is mostly used apart from some square-wave UMP simulations in Fig. 3. This is because sine-wave operation is usually used for servo drives where smoother and more vibration-free operation is required. Square-wave operation is more normal in power drives but the analysis below is also valid for square-wave operation.

\section{Machine Simulation: Surface Magnet Machine}

Dynamic eccentricity is where the rotor rotates off center so that there is a rotating UMP. In this section, we will investigate UMP at $80 \%$ (touch down level), 50\% (high fault level), $30 \%$ (fault developing), and 10\% (probable limit of manufacture tolerance) eccentricity. The simulations will be conducted on open circuit and with load—both motoring and generating. Unless otherwise stated, the starting point will be with the rotor moved horizontally to the right in the simulations and this ec-

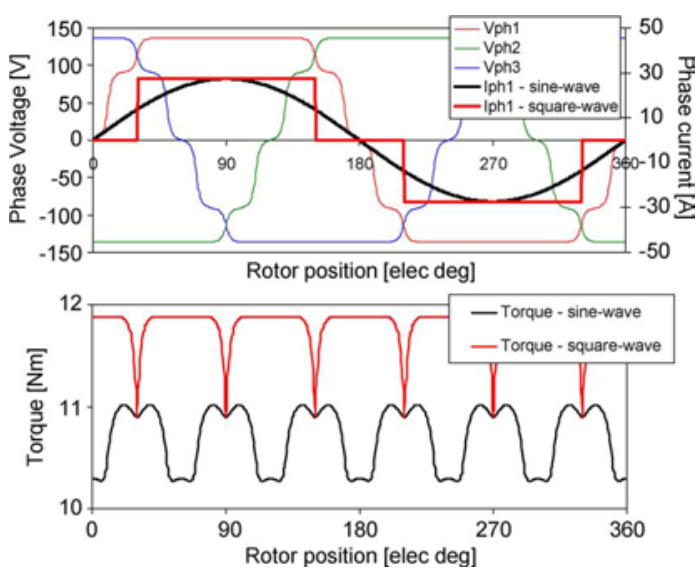

Fig. 2. Current, back-EMF, and torque simulations for basic machine.

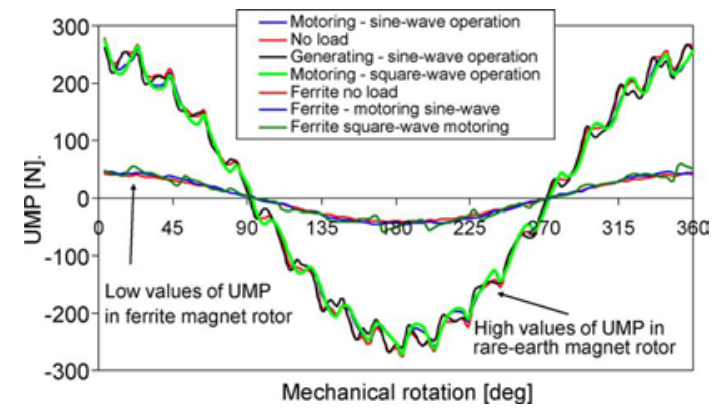

Fig. 3. Variation of UMP with no-load and full-load motoring and generating with sine-wave and motoring square-wave operation at $50 \%$ eccentricity.

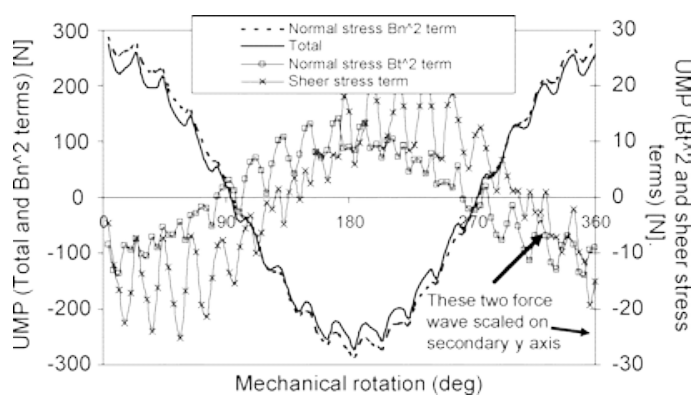

Fig. 4. UMP under load (sine-wave operation) at 50\% eccentricity-breakdown into stress terms (smaller components on right-hand side y-axis).

centricity then rotates with the rotor rotation. In addition, all the simulations consider the UMP along the horizontal $x$-axis. Because the eccentricity is dynamic, then the $y$-axis UMP is almost identical apart from a $90^{\circ}$ phase shift.

\section{A. Stress Components of UMP}

The UMP when there is $50 \%$ eccentricity (i.e., the rotor is $0.5 \mathrm{~mm}$ off center) is shown in Fig. 4. This illustrates that the tangential flux components do contribute some UMP which is just under $10 \%$ as found in [9]. In this simulation, the machine was fully loaded with $19.3 \mathrm{~A}$ on the rotor $q$-axis. In the following simulations, all the stress components were used.

\section{B. Load Variation: Sine-Wave and Square-Wave Operation}

The rotor eccentricity was maintained at $50 \%$, and the machine was simulated (sine-wave) with open circuit stator and 


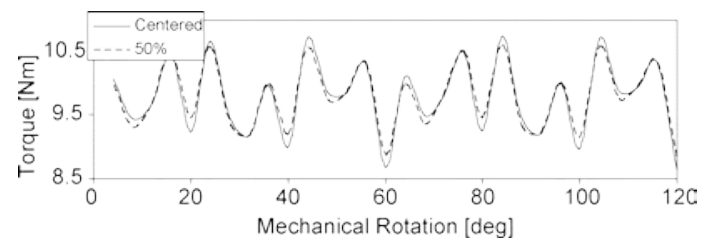

Fig. 5. Variation of sine-wave torque ripple when centered and with 50\% eccentricity (ripple from analytical torque calculation in Fig. 2 apparent).

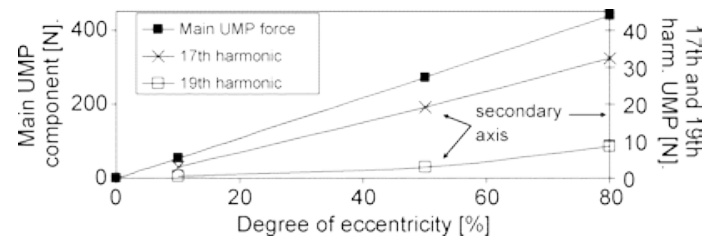

Fig. 6. UMP with eccentricity up to $80 \%$ and full load, 17 th and 19 th vibration harmonics included (scaled on right y-axis): sine-wave operation.

19.3 A on the $q$ and $q$-axis (motoring and generating). The results are shown in Fig. 3. It can be seen that the UMP is almost load independent. The magnets are thick and the peak flux density in the steel is about $1.7 \mathrm{~T}$ (i.e., on the knee of the B/H curve). Square-wave operation was simulated with the same peak current (Fig. 2); again this had little effect on the UMP. One point that should be considered is that the magnet appears as air to the stator MMF, and therefore, in terms of the length of air gap plus magnet, the per-unit eccentricity is much lower. To assess whether the rare-earth magnets suppress the UMP variation with load, then the magnet material was replaced with ferrite (which reduced the remanent flux density $B_{r}$ from 1.24 to $0.405 \mathrm{~T}$, the full-load torque from 10.7 to $3.4 \mathrm{Nm}$, and the peak tooth flux density from 1.7 to $0.7 \mathrm{~T}$ ). The results are given in Fig. 3 and it can be seen that the ferrite magnets reduce the UMP while there is still little load variation of the UMP, suggesting that it is the high effective air-gap length that suppresses the UMP due to loading.

\section{Torque Ripple (Cogging Torque)}

Rather than looking at open-circuit cogging torque, the torque ripple is investigated. The air-gap integrals use 360 points in the simulation and the rotor is stepped round with 4 movements. The torque ripple is shown in Fig. 5. There appears to be no noticeable variation in either mean torque or torque ripple at 50\% eccentricity. This is for the machine fully loaded and motoring.

\section{Variation of Peak UMP With Eccentricity}

The eccentricity varied from the centered position to $80 \%$ eccentricity with full load (rotor pullover and touchdown likely to occur). Fig. 6 shows the results; a very linear characteristic is observed. This is an interesting result. In [10], it was found that UMP increased more rapidly at higher eccentricity (above $50 \%$ ) for a cage induction motor (and verified experimentally). At $80 \%$ eccentricity, the UMP is over $400 \mathrm{~N}$ which is over ten times the machine weight in $\mathrm{Kg}$ force.

\section{E. Vibration Components}

In Fig. 3, it can be seen that there is ripple on the UMP. This could be used in a condition monitoring system to assess the health of the machine. Dynamic eccentricity produces a rotating UMP force vector at the machine speed, which at $6000 \mathrm{r} / \mathrm{min}$ is $100 \mathrm{~Hz}$. However, this could be confused with a mechanical

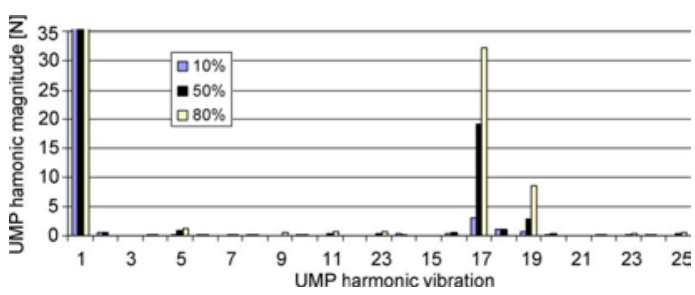

Fig. 7. Harmonic components of UMP with 10\%, 50\%, and 80\% eccentricity (first harmonics offscale): sine-wave operation.

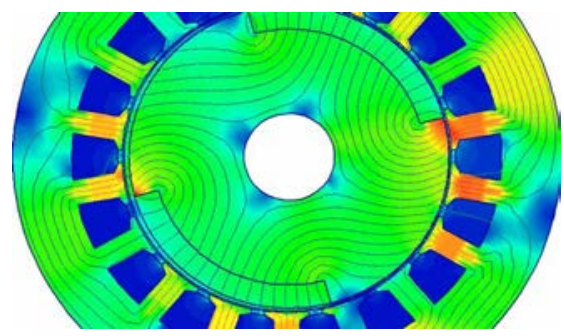

Fig. 8. Snapshot at start of simulations of consequent pole machine showing higher flux density on right; the rotor is rotated by $15^{\circ}$.

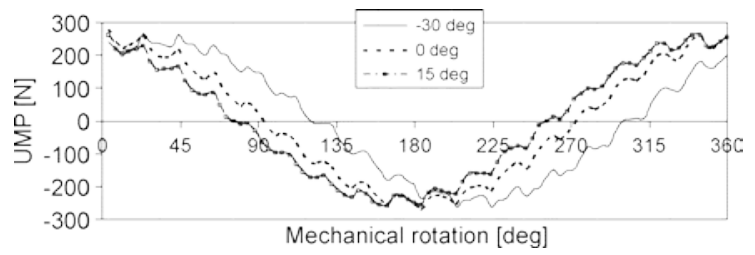

Fig. 9. UMP along $\mathrm{x}$-axis for different starting positions of $50 \%$ dynamic eccentricity (with respect to the $\mathrm{x}$-axis): sine-wave operation.

out-of-balance vibration. The ripple components of the UMP for this 18-slot machine are $18 \pm 1$ harmonics of the main rotating force wave (these are often called slot-passing vibrations). These are 1.7 and $1.9 \mathrm{kHz}$, and because they are UMP components, as opposed to tooth force ripple or nodal vibrations, they are likely to be more apparent during vibration monitoring [7]. The magnitudes are given in Fig. 6 while Fig. 7 gives the Fourier breakdown of the UMP vibrations at $10 \%, 50 \%$, and $80 \%$ eccentricity to highlight that these harmonics are the dominant UMP vibration components.

\section{F. UMP Variation With Position of Eccentricity}

With dynamic eccentricity, the point of minimum air gap (direction of eccentricity) is synchronized with the rotor. In all the simulations, the rotor was aligned at an angle of 15 to the horizontal; this is shown for the consequent machine in Fig. 8 and this alignment corresponds with the $q$-axis of phase 1 of the winding. In this section, simulations were carried out with the starting eccentricity at $-30^{\circ}$ (center of a magnet) and 15 (intermagnet or rotor $q$-axis). It can be seen in Fig. 9 that the UMP magnitudes are almost identical and the waveforms have a phase difference of $45^{\circ}$ due to the movement of the eccentricity at the starting point. If the eccentricity was static, then the UMP would manifest itself as a steady pull in the direction of minimum air gap with the slot-passing vibrations (as discussed above).

\section{G. Discussion}

The motor uses thick rare-earth magnets. It was found that the loading therefore had little effect on the UMP. If weaker mag- 


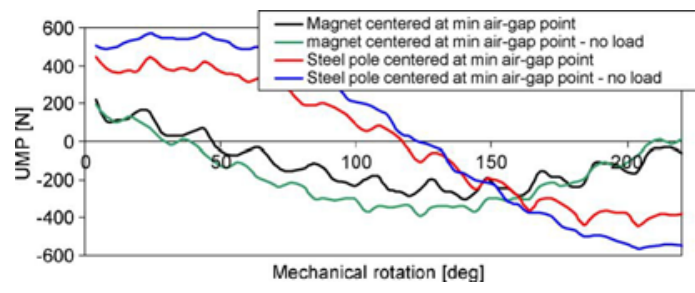

Fig. 10. UMP variation with $50 \%$ eccentricity (point of minimum air gap) at center of steel pole and center of magnet pole for consequent pole machine: sine-wave operation, load and no load.

nets (such as ferrite) or thinner magnets are used, then the effect of load would be more substantial as shown in [6] (although ferrite magnets did not affect the load dependence here because of the magnet thickness). The UMP is high although it is linear up to $80 \%$ eccentricity. The linear UMP/eccentricity characteristic means that the machine will be less susceptible to rotor pullover provided the frame, bearing mountings, and shaft are robust; in an induction motor, the UMP begins to rise almost exponentially when the UMP is more than 50\% [10]. The torque is not affected by the rotor eccentricity (unlike the switched reluctance machine in [11]).

\section{Consequent Pole Machine}

One solution to reduce the amount of magnet is to turn the machine into a consequent pole machine where alternate poles are steel (as an alternative to reducing the magnet thickness). Fig. 8 shows the flux plot at an instant in time when the consequent pole machine is loaded and this illustrates the arrangement. The torque reduces to $8.4 \mathrm{Nm}$. It can be seen that the flux density is higher on the right-hand side of the machine where there is minimum air gap (concentration of flux line in yoke).

Fig. 10 shows the UMP when the machine is loaded (sine-wave) and with 50\% eccentricity which is aligned with the magnet center and then with the steel pole center. It can be seen that the steel pole alignment produces higher UMP (445-N peak) compared to the magnet alignment (287-N peak), i.e., the dynamic eccentricity UMP peak magnitude is a function of the position of the eccentricity with respect to the rotor. This is in contrast to Fig. 9 where it is independent. This variation is because the steel pole allows the flux to concentrate in the center of the pole whereas the magnet produces a more even spread of flux around the point of minimum air gap. This generates higher UMP. The magnet-aligned UMP has about the same UMP magnitude as the surface magnet machine.

If the eccentricity was static, then there would be a steady pull of $(445+287) \div 2=366 \mathrm{~N}$ and a twice-rotational-speed vibration with peak-to-peak 445-287= $158 \mathrm{~N}$. Because the eccentricity is dynamic, then the ready rotating force vector magnitude varies with eccentricity position with respect to the rotor. In [6], static eccentricity was investigated for higher pole number machines and it was found that the UMP vibrations were very much a function of the machine topology.

Fig. 10 also gives the no-load UMP which is shown to be about $50 \%$ higher. This illustrates that for the consequent-pole machine the UMP is load dependent. In Section IV-B, it was stated that the large effective air gap reduced the load effect on UMP for the surface magnet machine. In the consequentpole machine, the steel poles greatly reduce the effective air-gap length and hence the UMP is now a function of load.

\section{CONCLUSION}

UMP in rare-earth magnet machines is high as shown in the results. Because of the strength and thickness of the magnets, the UMP was not found to be very load dependant in the surface-magnet rotor whereas it was load dependant for the consequent-pole rotor. The slot-passing frequency vibrations look appropriate for vibration monitoring since the magnitudes are almost linear with eccentricity. For the consequent-pole machine (with the dynamic eccentricity aligned with a steel pole center), the UMP was over 50\% higher than the surface-magnet machine arrangement.

\section{REFERENCES}

[1] A. Burakov and A. Arkkio, "Comparison of the unbalanced magnetic pull mitigation by the parallel paths in the stator and rotor windings," IEEE Trans. Magn., vol. 43, no. 12, pp. 4083-4088, 2007.

[2] R. Perers, U. Lundin, and M. Leijon, "Saturation effects on unbalanced magnetic pull in a hydroelectric generator with an eccentric rotor," IEEE Trans. Magn., vol. 43, no. 10, pp. 3884-3890, 2007.

[3] Z. Q. Zhu, D. Ishak, D. Howe, and J. Chen, "Unbalanced magnetic forces in permanent-magnet brushless machines with diametrically asymmetric phase windings,” IEEE Trans. Ind. Appl., vol. 43, no. 6, pp. 1544-1553, 2007.

[4] D. G. Dorrell, "The sources and characteristics of unbalanced magnetic pull in cage induction motors with either static or dynamic eccentricity," in Proc. Stockholm IEEE Power Technol., Stockholm, Jun. 1995, pp. 229-234.

[5] P. Vijayraghavan and R. Krishnan, "Noise in electric machines: A review,” IEEE Trans. Ind. Appl., vol. 35, no. 5, pp. 1007-1013, 1999.

[6] D. G. Dorrell, M. Popescu, C. Cossar, and D. Ionel, "Unbalanced magnetic pull in fractional-slot brushless PM motors," in Proc. IEEE IAS Annual Meeting, Edmonton, Canada, Oct. 2008, pp. 1-8.

[7] D. G. Dorrell and C. Cossar, "A vibration-based condition monitoring system for switched reluctance machine rotor eccentricity detection," IEEE Trans Magn., vol. 44, no. 9, pp. 2204-2214, Sep. 2008.

[8] A. C. Smith and D. G. Dorrell, "The calculation and measurement of unbalanced magnetic pull in cage induction motors with eccentric rotors. Part 1: Analytical model," Proc. Inst. Electr. Eng. Electric Power Appl., vol. 143, no. 3, pp. 193-201, 1996.

[9] K. J. Binns and M. Dye, "Identification of principal factors causing unbalanced magnetic pull in cage induction motors," Proc. Inst. Electr. Eng., vol. 120, pp. 349-354, 1973.

[10] D. G. Dorrell and A. C. Smith, "The calculation and measurement of unbalanced magnetic pull in cage induction motors with eccentric rotors. Part 2: Experimental verification,” Proc. Inst. Electr. Eng. Electric Power Appl., vol. 143, no. 3, pp. 202-210, 1996.

[11] D. G. Dorrell, I. Chendurza, and C. Cossar, "Effects of rotor eccentricity on torque in switched reluctance machines," IEEE Trans. Magn., vol. 41, no. 10, pp. 3961-3963, Oct. 2005.

[12] L. Wang, R. W. Cheung, Z. Ma, J. Ruan, and Y. Peng, "Finite-element analysis of unbalanced magnetic pull in a large hydro-generator under practical operations,” IEEE Trans. Magn., vol. 44, no. 6, pp. 1558-1561, Jun. 2008.

[13] P. Frauman, A. Burakov, and A. Arkkio, "Effects of the slot harmonics on the unbalanced magnetic pull in an induction motor with an eccentric rotor," IEEE Trans. Magn., vol. 43, no. 8, pp. 3441-3444, Aug. 2007.

[14] M. J. DeBortoli, S. J. Salon, D. W. Burow, and C. J. Slavik, "Effects of rotor eccentricity and parallel windings on induction machine behavior: A study using finite element analysis,"” IEEE Trans. Magn., vol. 29, no. 2, pp. 1676-1682, Mar. 1993.

[15] C. Bi, Z. J. Liu, and T. S. Low, "Effects of unbalanced magnetic pull in spindle motors," IEEE Trans. Magn., vol. 33, no. 5, pp. 4080-4082, Sep. 1997.

[16] I. Husain, A. Radun, and J. Nairus, "Unbalanced force calculation in switched-reluctance machines,” IEEE Trans. Magn., vol. 36, no. 1, pt. 2, pp. 330-338, Jan. 2000.

[17] J. T. Li, Z. J. Liu, and L. H. A. Nay, "Effect of radial magnetic forces in permanent magnet motors with rotor eccentricity,” IEEE Trans. Magn., vol. 43, no. 6, pp. 2525-2527, Jun. 2007. 